\title{
RELIABILITY OF THE 20-METRE SHUTTLE RUN FOR CHILDREN WITH INTELLECTUAL DISABILITIES
}

\begin{abstract}
Mike Gillespie
University of Northampton, Northampton, UK

Individuals with intellectual disabilities (ID) typically exhibit lower levels of cardiovascular fitness as compared to their non-disabled peers. Identification of valid, reliable indicators of fitness for this group is critical in terms of developing and evaluating interventions aimed at ameliorating deficits in fitness. While significant literature is available regarding fitness tests among adults with ID, less is known about appropriate assessments for children with ID. The purpose of the current investigation is to examine the reliability of the 20-metre shuttle run test for young children with ID. Thirty children (mean age $=8$ years, zero months) with ID completed two 20-metre shuttle runs 72 hours apart. Results indicated a test-retest reliability of $R=.53$, which is lower than observed in previous investigations. Discussion as to the findings and application of results for practitioners are addressed.
\end{abstract}

KEYWORDS: Intellectual disability, cardiovascular fitness, test reliability, shuttle run test.

\section{INTRODUCTION}

Significant evidence exists indicating that the cardiovascular fitness of children with intellectual disabilities (ID) is significantly substandard to that of their non-disabled peers (Fernhall et al., 1998; Fernhall, Millar, Pitetti, Hensen \& Vukovich, 2000; Gillespie, 2003; Pitetti, Yarmer \& Fernhall, 2001). While evidence regarding the physical activity levels of children with ID appears to be inconclusive (Frey, Stanish \& Temple, 2008), literature concerning fitness levels among this group indicates deficits that have the potential to impact negatively on individuals from a health perspective.

A number of factors have been posited to explain low levels of physical fitness observed among children with ID. In addition to potential comorbid impairments (e.g. Down Syndrome) that may impact function, lack of opportunity (Cluphf, O' Connor \& Vanin, 2001), inaccessible facilities and organizations (Rimmer, 1994), lack of motivation (Frey, McCubbin, HanniganDowns, S., Kasser \& Skaggs, 1999; Pitetti, Millar \& Fernhall, 2000), heterogeneity of participants (Baumgartner \& Horvat, 1991), and physical inactivity (Faison-Hodge \& Porretta, 2004; Frey et al., 1999; Obruskinova, Valkova, \& Block, 2003; Sit, McKenzie, Lian \& McManus, 2008) have all been cited as potential factors behind poor fitness levels of children with ID. Additionally, issues associated with task understanding have been identified as a mitigating factor in the collection of valid, reliable fitness data for persons with ID (Baumgartner \& Horvat, 1991; Fernhall, 1992; Frey et al., 1999; Pitetti et al., 2000).

Identification of rigorous, valid indicators of fitness that can be employed for use with children with ID appears crucial in order that accurate measures can be obtained during assessment. In spite of the substantial body of literature devoted to cardiovascular fitness levels among persons with ID, there are few valid and reliable tests evidenced for this group (Seidl, 1988). McCubbin, Rintala and Frey (1997) note five field tests (1.0 mile walk/run, 1/5 mile run, Schwinn Air-Dyne bicycle ergometer, modified Canadian Step Test, modified Leger and Lambert shuttle run) that have been validated with respect to ascertaining aerobic fitness for adults with ID. 
While the above tests have been identified as valid indicators of cardiovascular fitness for adults with ID, scant evidence exists concerning the appropriateness of aerobic field tests for young children with ID. The 20metre (20-m) shuttle run (Leger \& Lambert, 1982) has been cited as a reliable field test for children with ID (Fernhall et al., 1998; Pitetti, Yarmer \& Fernhall, 2001). However, minimal examination extending this line of inquiry has been conducted in order to make more conclusive determinations as to the appropriateness of the 20-m shuttle run with younger children with ID. Seidl, Reid and Montgomery (1987) note that when assessing fitness levels of persons with ID, validity of test protocols commonly proves to be specific to the level of ID, age or sex of the persons being tested.

Identification of valid, reliable field tests is particularly important given the need to promote cardiovascular fitness and physical activity among children with ID, and to ascertain their present level of function in order that appropriate intervention can be undertaken where appropriate. The dearth of valid, reliable measurement instruments is likely in part responsible for children with ID being both the least investigated and understood group concerning cardiovascular fitness (Fernhall, Tymeson \& Webster, 1988), with further investigation into this cohort having been identified as a critical area of research (Chanias, Reid \& Hoover, 1998).

The purpose of the present investigation is to examine the reliability of the $20-\mathrm{m}$ shuttle run test for young children with ID. Given that many poor habits associated with disease risk (e.g. obesity) often occur early in life (Blair et al., 1989; Fernhall et al., 2000), development of understanding regarding current levels of cardiovascular fitness among young individuals with ID is crucial in order that the benefits of cardiovascular fitness to children with ID can be espoused as early as possible.

\section{METHOD}

\section{Participants}

Thirty students (15 male, 15 female) enrolled in an urban public school system were recruited as participants for the study. Mean age of participants was eight years, zero months (SD $=7.60$ months; range 80-109 months). Participants came from four different schools within the district, with classroom placement for all individuals being a separate alternative classroom. Convenience sampling was used to recruit participants.

All of the individuals were identified as having a mild level of ID according to school district identification procedures. Specific information concerning educational and adaptive behaviour test scores were not made available; however, school district policies identified students with mild levels of ID as having an IQ score between 55-70 and deficits in adaptive skill behaviours. Concomitant impairments were not identified among any of the participants. Written informed consent was acquired from parents/guardians. Ethical approval was obtained from both the researcher's University and the school district where the participants were enrolled as students.

\section{Testing Protocol}

The 20-m shuttle run (Leger \& Lambert, 1982) was selected as the cardiovascular fitness protocol for the current study. While the one-mile walk-run test appears to be the most commonly used field test among children (Fernhall et al., 2000), the 20-m was selected because of the relatively easy administration procedures and the ease of performance for children with ID (Fernhall et al., 1998; 2000), and has proven to be reliable across a variety of groups (Guerra, Pitetti \& Fernhall, 2003; MacDonncha, Watson, McSweeney \& O'Donovan, 1999). The 20-m run also provides a field test that can be administered by individuals providing physical activity programs to children with ID (e.g. physical education teachers, coaches) in a reasonably small amount of space, which is critical given spatial constraints often 
observed in school and community-based facilities.

\section{Procedures}

All participants were tested individually. Testing took place in the gymnasia of the four school buildings. The $20-\mathrm{m}$ course was marked by two strips of tape at opposite ends of the course, with a pylon being placed at each end of the pieces of tape. Participants were told that the goal of the activity was to run between the two pieces of tape on the floor before the "beep went off on the cd". Pace music was played from a compact disc recording. The protocol required participants to run the 20 metres at a speed of 8.5 kilometres per hour during the first minute of each test, with a subsequent increase in running speed of 0.5 kilometres per hour each minute. An inability to keep up with the required pace for two consecutive laps resulted in protocol termination. The test was also terminated if the participant was physically unable to continue (e.g. through fatigue, voluntary cessation).

Participants were provided with practice sessions before data collection sessions took place. These sessions were designed to orient participants to the task. The use of practice sessions has been found to neutralise lack of experience in performing field tests of cardiovascular fitness for participants with ID (Cressler, Lavay \& Giese, 1988; Pitetti et al., 2001). During the orientation sessions, participants were a) shown the test environment, b) allowed to listen to the pace music, and c) practice the $20-\mathrm{m}$ shuttle run protocol that would be administered during data collection. A pacer ran alongside the participant during the practice lengths for motivational purposes.

Upon completion of the orientation sessions, participants were tested on the $20-\mathrm{m}$ shuttle run during two separate sessions that took place 72 hours apart. The first testing session took place 72 hours after the last orientation session. During the two test sessions, participants entered the testing area and were briefly re-oriented to the shuttle run. This re-orientation consisted of a verbal explanation of the test and a two-length demonstration by the researcher. The participant was then asked to complete the 20$\mathrm{m}$ test. As in the orientation sessions, a pacer ran along with the participant for motivational purposes. The score for both shuttle runs was the number of laps (i.e. one lap consisted of running from one length of the tape to the other) completed by the participant.

\section{Statistical analysis}

Data were analysed using an intraclass correlation coefficient. Intraclass correlation is the preferred method of examining testretest data because of the ability to analyse repeated measures data on the same variable (MacDonncha et al., 1999; Vincent, 1999). Descriptive statistics (i.e. means and standard deviations) were also calculated for each of the two testing sessions.

\section{RESULTS}

The mean number of laps completed on the first trial of the $20-\mathrm{m}$ run was 8.43 ( $\mathrm{SD}=$ 3.11; Range $=3-19)$. Participants performed an average of 8.13 laps $(\mathrm{SD}=3.90$; Range $=$ 1-20) on the second attempt. Results of the intraclass correlation indicated a test-retest reliability coefficient of $\mathrm{R}=.53$ between scores on the first and second $20-\mathrm{m}$ shuttle runs.

\section{DISCUSSION}

Results in the present study concerning reliability differed from those previously cited in the literature. Numerous investigations (e.g. Fernhall et al., 2000; MacDonncha et al., 1999; Pitetti et al., 2000) have evidenced high intraclass reliability coefficients for persons with ID on a variety of cardiovascular fitness tests. For example, Fernhall et al. (2000) found an intraclass coefficient of $\mathrm{R}=.94$ on the 20-m shuttle run for children and adolescents with ID, and MacDonncha et al. (1999) also revealed $\mathrm{R}=.94$ for adolescents. These findings starkly contrast with those in the current study.

Several possible explanations may account for the relatively low reliability found 
in the present investigation. The age of participants may have accounted for the low correlation between trials of the 20-m shuttle run. Participants in the present study ranged in age from six years, six months to nine years, one month, with a mean age of eight years zero months. Analysis of previous studies examining cardiovascular fitness testing among children with ID reveals the use of participants who were older than those in the current study. For example, Fernhall et al. (2000) used participants with a mean age of 13.7 years, and Pitetti et al. (2000) had a participant pool with a mean age of 13.6 years. An investigation by Pitetti et al. (2001) used participants with ages closer in range (eight years, eight months for males; nine years, zero months for females) to those in the present study; however, intraclass correlation coefficients were not provided. Although participants similar to the mean age in the present study have been investigated previously, perhaps the test protocol is inappropriate for those in the younger portion of the cohort in the current study. It is also possible that the age range of participants in the current study may have impacted on testretest reliability; however, similar ranges in participant age have been evidenced previously in the literature (MacDonncha et al., 1999; Ozmen, Un Yildirim, Yuktasir \& Beets, 2007). Further investigation utilizing participant groups with a narrower age range may be warranted.

Suitability of the $20-\mathrm{m}$ protocol for some of the younger participants in the current investigation may have contributed to the reliability levels observed. While the speed and distance employed in the current investigation mirror those used in previous investigations (Fernhall et al., 1998; Pitetti et al., 2001), because of the young age of some of the participants this protocol may not have been suitable for these individuals. Further investigation into the suitability of this protocol, or modifications in speed and/or distance, is recommended.

The young age of participants in the present study may have also combined with issues pertaining to participant motivation.
Motivation of individuals with ID to perform "best effort" has been cited as a justificatory cause in terms of both poor performance on fitness tests (Fernhall \& Tymeson, 1987; Pitetti et al., 2000) and low levels of cardiovascular fitness (Frey et al., 1999). Motivational concerns when evaluating physical fitness of persons with ID are exacerbated when testing children and adolescents, which may produce data that are not reliable (Pitetti et al., 2000). Given the young age of participants in the present investigation, steps were taken to try to increase participant motivation. For example, participants were provided with a tangible reinforcer (i.e. a sticker) upon completion of both 20-m shuttle runs. Additionally, a pacer was utilized to increase effort. This strategy of pacer utilization has been successful in producing results with high reliability in previous investigations (e.g. Fernhall et al., 2000). Previous research had participants with ID perform in small groups along with the pacer, while the current investigation had participants execute the shuttle run in a oneto-one setting along with the pacer. The technique employed in the present study was utilized because it was thought that the presence of additional individuals may have served to distract participants given the young age within the cohort. The original 20-m protocol (Leger \& Lambert, 1982) was designed for group administration, so the selection of an individual testing situation in the present study may have impacted upon reliability. Previous studies that have employed the 20-m protocol on children with ID have used groups as small as two participants (Guerra et al., 2003; Ozmen et al., 2007); however, performing with peers may have in fact served as a motivator that could potentially have yielded higher degrees of reliability than those evidenced. Future investigations involving young participants with ID should examine the reliability and validity of testing individually versus group settings.

Familiarization procedures may have combined with the low age of participants to produce low intraclass correlations on the 20- 
$\mathrm{m}$ shuttle run. The need to orient participants with ID prior to performing fitness assessments has been well documented in the literature (Cressler et al., 1988; Fernhall \& Tymeson, 1987; Seidl, 1998). In the present investigation, measures were taken to ensure that participants were familiar with the procedures associated with the shuttle run. The protocol of these practice sessions was similar to those previously shown to be successful in maximizing the likelihood of achieving reliable results among individuals with ID (Pitetti et al., 2001). However, participants in the current study did not exhibit high degrees of test-retest reliability. As mentioned previously, participants in the current study were younger than those recruited in previous research. Perhaps the familiarization procedures employed were insufficient to orient the young participants in this study. While the practice sessions used appeared to be of an appropriate quantity based on previous research, it is possible that more familiarization trials/sessions were necessary to produce greater degrees of reliability on the shuttle run. Subsequent research focused on the provision of additional familiarization trials for young participants with ID appears warranted.

Results of the present investigation indicate that the 20-m shuttle run may not be a reliable measure of cardiovascular fitness for young children with ID. It may be that the variable of interest in terms of assessing young children with ID may be physical activity, rather than fitness, levels. While recognizing that there is a need for valid, reliable indicators of physical fitness for reasons posited earlier, if further investigation indicates that such instruments are not available because of some of the factors addressed in the discussion (e.g. motivation, suitability of protocol, familiarization) it may be that physical activity monitoring may be the most appropriate measure. There is a significant dearth of literature in terms of physical activity levels among youth with ID (Frey et al., 2008), suggesting that further research is necessary, particularly in the context of findings from the present study.
While recognising the limitations in generalising the current results to the wider population of children with ID, findings from the current investigation have implications from the perspective of practitioners working in applied settings. Individuals such as teachers and coaches should exercise caution when interpreting the results of shuttle run tests for students and athletes in this group, and for using such results to make decisions regarding program development, implementation and evaluation. Examination as to the suitability of modified protocols with respect to speed and/or distance may be appropriate to explore in applied settings. Additionally, such professionals can work collaboratively with researchers in order to collect further empirical data so that valid, reliable indicators of physical fitness among this group can be identified. It appears that further investigation into the reliability and validity of the shuttle run, as well as other field-based cardiovascular fitness protocols, for younger participants with ID is necessary.

\section{REFERENCES}

Baumgartner, T., \& Horvat, M. (1991). Reliability of field-based cardiovascular fitness running tests for individuals with mental retardation. Adapted Physical Activity Quarterly, 8, 107-114.

Blair, S., Kohl, H., Paffenbarger, R., Clark, D., Cooper, K., \& Gibbons, L. (1989). Physical activity and all cause mortality: A prospective study of healthy men and women. Journal of the American Medical Association, 262, 2395-2401.

Chanias, A., Reid, G., \& Hoover, M. (1998). Exercise effects on health-related physical fitness of individuals with an intellectual disability: A meta-analysis. Adapted Physical Activity Quarterly, 15, 119-140.

Cluphf, D., O'Connor, J., \& Vanin, S. (2001). Effects of aerobic dance on the cardiovascular endurance of adults with intellectual disabilities. Adapted Physical Activity Quarterly, 18, 60-71.

Cressler, M., Lavay, B., \& Giese, M. (1988). The reliability of four measures of 
cardiovascular fitness with mentally retarded adults. Adapted Physical Activity Quarterly, 5, 285-292.

Faison-Hodge, J., \& Porretta, D. (2004). Physical activity levels of students with mental retardation and students without disabilities. Adapted Physical Activity Quarterly, 21, 139-152.

Fernhall, B. (1992). Physical fitness and exercise testing of individuals with mental retardation. Medicine and Science in Sports and Exercise, 25, 442-450.

Fernhall, B., Millar, A., Pitetti, K., Hensen, T., \& Vuckovich, M. (2000). Cross validation of the 20-metre shuttle run test for children with mental retardation. Adapted Physical Activity Quarterly, 17, 402-412.

Fernhall, B., Pitetti, K., Vuckovich, M., Stubbs, N., Hensen, T., Winnick, J., \& Short, F. (1998). Validation of cardiovascular fitness field tests in children with mental retardation. American Journal on Mental Retardation, 102, 606-612.

Fernhall, B., \& Tymeson, G. (1988). Validation of a cardiovascular fitness field test for adults with mental retardation. Adapted Physical Activity Quarterly, 5, 4959.

Fernhall, B., Tymeson, G., \& Webster, G. (1988). Cardiovascular fitness of mentally retarded individuals. Adapted Physical Activity Quarterly, 5, 12-18.

Fernhall, B., \& Tymeson, G. (1987). Graded exercise testing of mentally retarded adults: A study of feasibility. Archives of Physical Medicine and Rehabilitation, 68, 363-365.

Frey, G., McCubbin, J., Hannigan-Downs, S., Kasser, S., \& Skaggs, S. (1999). Physical fitness of trained runners with and without mild mental retardation. Adapted Physical Activity Quarterly, 16, 126-137.

Frey, G., Stanish, H., \& Temple, V. (2008). Physical activity of youth with intellectual disability: Review and research agenda. Adapted Physical Activity Quarterly, 25, 95117.

Gillespie, M. (2003). Cardiovascular fitness of young Canadian children with and without mental retardation. Education and
Training in Developmental Disabilities, 38, 296-301.

Guerra, M., Pitetti, K., \& Fernhall, B. (2003). Cross validation of the 20-meter shuttle run for adolescents with Down Syndrome. Adapted Physical Activity Quarterly, 20, 7079.

Leger, L., \& Lambert, J. (1982). A maximal multistage 20-metre shuttle run test to predict $\mathrm{VO}_{2 \max }$. European Journal of Applied Physiology and Occupational Physiology, 49, 1-12.

MacDonncha, C., Watson, A., McSweeney, T., \& O'Donovan, D. (1999). Reliability of Eurofit physical fitness items for adolescent males with and without mental retardation. Adapted Physical Activity Quarterly, 16, 8695.

McCubbin, J., Rintala, P., \& Frey, G. (1997). Correlational study of three cardiorespiratory field tests for men with mental retardation. Adapted Physical Activity Quarterly, 14, 43-50.

Obruskinova, I., Valkova, H., \& Block, M. (2003). Impact of inclusion in general physical education on students without disabilities. Adapted Physical Activity Quarterly, 20, 230-245.

Ozmen, T., Un Yildirim, N., Yuktasir, B, \& Beets, M. (2007). Effects of school-based cardiovascular fitness training in children with mental retardation. Pediatric Exercise Science, 19, 171-178.

Pitetti, K., Yarmer, D., \& Fernhall, B. (2001). Cardiovascular fitness and body composition of youth with and without mental retardation. Adapted Physical Activity Quarterly, 18, 127-141.

Pitetti, K., Millar, A., \& Fernhall, B. (2000). Reliability of a peak performance treadmill test for children and adolescents with and without mental retardation. Adapted Physical Activity Quarterly, 17, 322-332.

Rimmer, J. (1994). Fitness and rehabilitation programs for special populations. Dubuque: Brown \& Benchmark.

Seidl, C. (1988). Considerations for fitness appraisal, programming and counselling of people with intellectual disabilities. 
Canadian Journal of Applied Physiology, 23, 185-211.

Seidl, C., Reid, G., \& Montgomery, G. (1987). A critique of cardiovascular fitness testing with mentally retarded persons. Adapted Physical Activity Quarterly, 4, 106116.

Sit, C., McKenzie, T., Lian, J., \& McManus, A. (2008). Activity levels during physical education and recess in two special schools for children with mild intellectual disabilities. Adapted Physical Activity Quarterly, 25, 247-259.

Vincent, W. (1999). Statistics in Kinesiology $\left(2^{\text {nd }} E d.\right)$. Champaign, IL: Human Kinetics.

\section{RELIABILITÄT DES 20-METER PENDELLAUFS FÜR KINDER MIT INTELLEKTUELLER BEHINDERUNG}

(Resümee)

Personen mit intellektueller Behinderung (IB) zeigen typischerweise ein niedrigeres Niveau an kardiovaskulärer Fitness als nichtbehinderte Gleichaltrige. Die Festlegung von validen und reliablen Fitness-Indikatoren für diese Gruppe ist kritisch in Hinblick auf die Entwicklung und Evaluierung von Interventionen, die eine Verbesserung des Fitness-Defizits beabsichtigen. Während aussagekräftige Literatur bezüglich Fitnesstests für Erwachsene mit IB verfügbar ist, ist über geeignete Assessment-Verfahren für Kinder mit IB weniger bekannt. Die Absicht der aktuellen Untersuchung ist es, die Reliabilität des 20-Meter Pendellauf-Tests für Kinder mit IB zu überprüfen. Dreißig Kinder (Durchschnittsalter $=8$ Jahre, 0 Monate) mit IB absolvierten zwei 20Meter Pendelläufe im Abstand von 72 Stunden. Die Ergebnisse zeigten eine Test-Retest-Reliabilität von $R=.53$, was ein niedrigerer Wert ist als er in früheren Untersuchungen beobachtet wurde. In der Diskussion werden die Ergebnisse und die Anwendung der Resultate für die Praxis angesprochen.

SCHLÜSSELWÖRTER: Intellektuelle Behinderung, Kardiovaskuläre Fitness, Test-Reliabilität, Pendellauf-Test.

\section{LA FIABILITE DU TEST NAVETTE DE 20 METRES POUR DES ENFANTS DEFICIENTS INTELLECTUELLES}

(Résumé)

Les individus déficients intellectuels (DI) démontrent typiquement une condition physique inférieure par rapport à leurs collègues non handicapés. L'identification d'indicateurs de la condition physique valides et fiables est indispensable pour le développement et l'évaluation d'interventions visant à améliorer les déficits de condition physique. Bien qu'il existe suffisamment d'études concernant les tests de condition physique chez des adultes DI, peu d'études examinent les évaluations appropriées pour des enfants avec DI. L'objectif de cette étude est d'évaluer la fiabilité du test navette de 20 mètres chez des enfants DI. Trente enfants (age moyen $=8$ ans, 0 mois) DI ont complété deux tests navette de 20 mètres à un intervalle de 72 heures. Les résultats indiquent une fiabilité test-retest de $\mathrm{R}=0,53$, ce qui est inférieur à ce qui a été observé dans des études précédentes. Une discussion concernant les résultats et leur application par des praticiens est menée.

MOTS CLEFS: déficience intellectuelle, condition physique, fiabilité du test, test navette. 